\title{
Electron and Phase-contrast Microscopy of Spores in Two Species of the Genus Mycotypha (Mucorales)
}

\author{
By T. W. K. YOUNG \\ Botany Departments, Chelsea College, and Birkbeck College, \\ University of London
}

(Accepted for publication 27 September 1968)

\begin{abstract}
SUMMAR Y
Capitella of Mycotypha africana and $M$. microspora regularly produce sterigmata of two lengths. Spores of $M$. africana are dimorphic and this is probably also the case in $M$. microspora. A membrane which separates from the spore, particularly on germination, is interpreted as evidence that the spore of M. africana is a sporangiole and it is suggested that the genus Mycotypha should probably be classified in Thamnidiaceae.
\end{abstract}

\section{INTRODUCTION}

Fenner (1932) described the asexual stage of a mucoraceous fungus Mycotypha microspora Fenner and classified it in Mucoraceae, as defined by Gaumann (I928). Since conidia are the only spores known to be produced in the asexual stage, Hesseltine (1955) suggested that $M$. microspora should probably be included in Cunninghamellaceae. Novak \& Backus (1963) described a second species, $M$. africana Novak \& Backus, reported to produce zygospores which are typically mucoraceous in form. In both species, asexual spores are produced on sterigmata which develop from the capitellum of the conidiophore. Spores are dry and readily detached at maturity. Liberated spores of $M$. microspora are described as varying from ovoid to spherical (Fenner, 1932) and those of $M$. africana as oblong-elliptic and globose to oval (Novak \& Backus, 1963).

There are clearly two forms of spore in Mycotypha africana and there appear to be two spore forms in $M$. microspora also. The aim of this paper is to describe features of interest associated with spore dimorphism in the genus Mycotypha as revealed by electron and phase-contrast microscopy and, in the light of these findings, to discuss the possible re-classification of this genus as a member of Thamnidiaceae.

\section{METHODS}

The strain of Mycotypha africana used was obtained from the Centraalbureau voor Schimmelcultures, Baarn, and M. microspora from Birkbeck College, London. They were grown on malt and potato-glucose agars either at $25^{\circ}$ or room temperature. Germinating spores of $M$. africana were obtained by incubating them at $37^{\circ}$ for about ro hr.

Single-stage carbon replicas were prepared by the method used previously (Young, 1968). Electron micrographs were taken on Zeiss E.M. 9 and Hitachi I I A microscopes. Anoptral phase-contrast and light photographs were taken with a Reichert Zetopan microscope. 


\section{RESULTS}

A capitellum of Mycotypha microspora subjected directly to an intense beam of electrons may split longitudinally along the surface exposed to the beam, unfold and flatten, thus exposing the inner surface (Pl. 2, fig. 17). Apertures of two sizes can be seen 'large' and 'small' which alternate if traced in a shallow helix around the capitellum (Fenner's shallow primary spiral). Fenner (1932) observed the helical arrangement of conidia on the capitellum and noted that those on one turn alternate with those on the turn directly above and below it. On a collapsed capitellum they appear to be arranged in diagonal rows. If the apertures are traced diagonally, a row of 'large' holes alternates with a row of 'small' ones. To consider only the larger apertures, it can be seen that they alternate in the manner described by Fenner. This is also true of the smaller apertures considered in isolation from the larger ones.

Persistent sterigmata protrude from the external surface of the capitellum. These are cone-shaped, either 'long' or 'short', and alternate as described above. Thus, sterigmata are dimorphic. At the region of attachment of the spore stalk to the sterigma is an annular constriction (Pl. 2, fig. 17). This is a weak zone and the mature spore is readily detached here. An approximately circular scar can be observed at the apex of a sterigma and at the base of a detached spore (Pl. 2, fig. I4, 16). Between the sterigmata are minute spines which are just visible with the light microscope in $M$. africana (Pl. 3, fig. 2r) and clearly shown in replicas (Pl. r, fig. I, 4).

Detached spores of Mycotypha microspora, as seen in silhouette, are smooth. Three shapes are common in profile; oval with a stalk, approximately rectangular with or without a stalk and spherical (Pl. I, fig. I0). In replicas the spherical spore tends to lie with the stalk either upwards (Pl. 2, fig. I4) or downwards (Pl. 2, fig. I5). The oval spore tends to lie with the stalk horizontal (P1. 2, fig. I2). A spore in replica invariably shows a single large indentation which may be due to drying out during processing. Spores are probably observed in a collapsed state and may not actually be biconcave. Thus those which appear rectangular in profile could represent collapsed spheres (P1. 2, fig. I3).

The surface of the spore as seen in replica is granular or roughened and no pattern of surface structure is evident. This contrasts with other described members of Cunninghamellaceae. The pyramidal conidial spines of Cunninghamella elegans Lendner have raised, angular bases and conidia of Phascolomyces articulosus Boedijn are covered with numerous flattened projections. However, ultrastructural spore morphology of Mycotypha microspora, as seen in profile and replica, closely resembles that of Cokeromyces poitrasii Benjamin in Thamnidiaceae.

Detached spores of Mycotypha africana are either oval (Pl. 2, fig. 18) or spherical (Pl. 2, fig. I I). Liberated spores of $M$. microspora tend to be either oval (Pl. 2, fig. I2) or spherical (P1. 2, fig. I6). However, this difference is not so sharply defined as in $M$. africana. The oval spores of $M$. africana are separable from those of $M$. microspora since the latter bear relatively prominent stalks whilst the former do not. Further, there can often be distinguished towards the base of the spore in $M$. africana a roughly circular, raised zone (Pl. 2, fig. 18,19$)$. This distinctive mark has not been observed on the oval spores of $M$. microspora.

The smaller spherical spores can be distinguished on a similar basis. The stalk in spores of both species is relatively short. However, there can often be observed either 
one or two raised zones on the spores of Mycotypha africana (Pl. 2, fig. II) which are not evident on those of $M$. microspora (Pl. 2, fig. 15). A further difference between the two species involves the appearance of liberated spores observed in a mass. Those of $M$. africana often fail to separate and can be seen as chains in which oval spores alternate with spherical ones (Pl. I, fig. 6, 7). Groups of two or three spores are common. Liberated spores of $M$. microspora do not appear to adhere in chains. Detached spores of $M$. africana and $M$. microspora are compared diagrammatically in Fig. I.

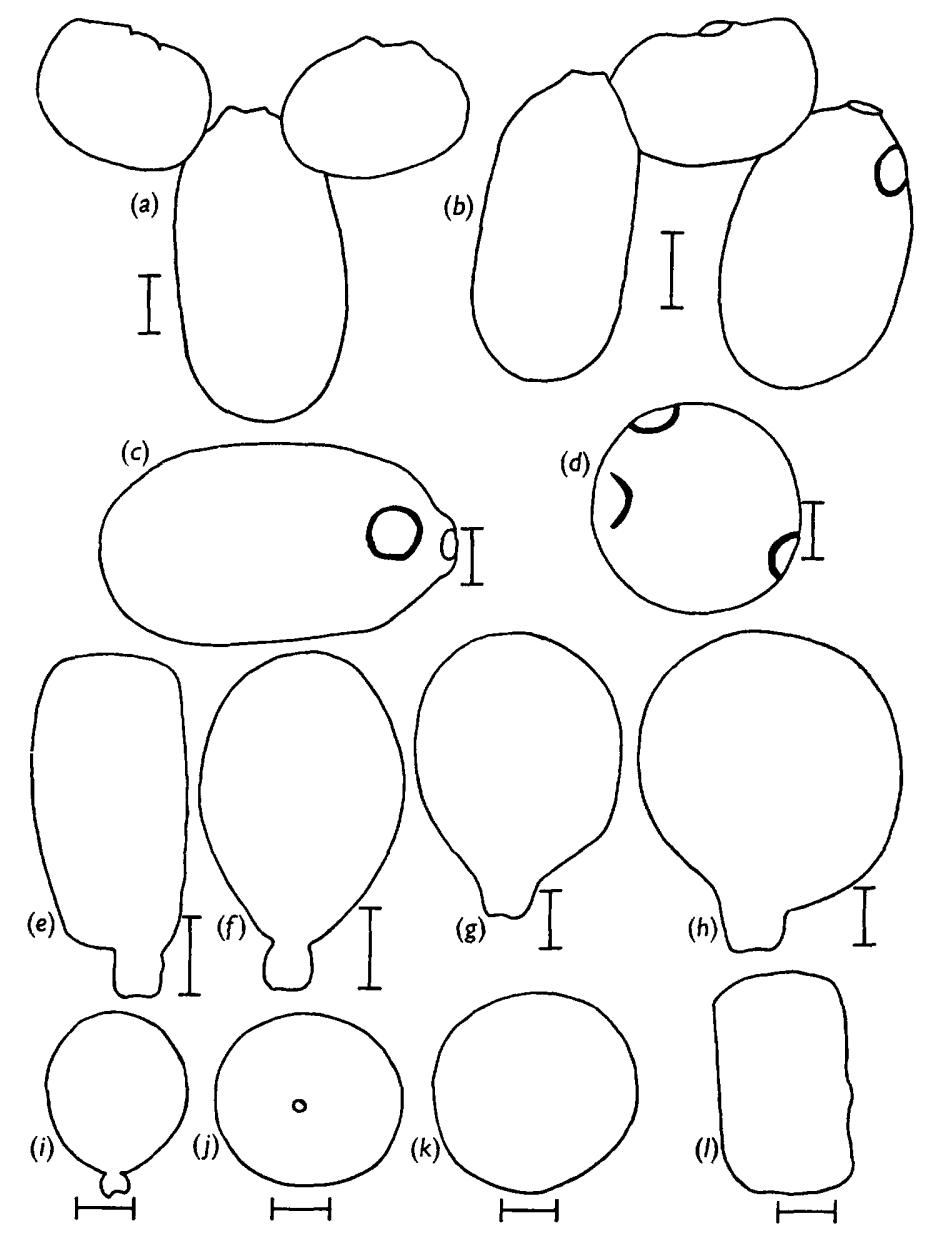

Fig. I. The scale line indicates I $\mu a-d$, Mycotypha africana: outlines of spores traced from photographs of replicas; they are clearly dimorphic. $e-l, M$. microspora: spore outlines for comparison with those of $M$. africana; there is a greater range of form although they tend to be either oval or spherical.

It seems highly probable that raised zones on the spores of Mycotypha africana correspond to areas of contact between adjacent spores on the capitellum and in detached chains. The positions of these areas support this view (Fig. I $a$ to $d$ ). Spherical spores attached to the capitellum (Pl. 3, fig. 20) fit between oval ones and contact the 
latter on the basal curve of the oval. The apparent absence of raised zones on the spores of $M$. microspora could be explained by assuming that relatively long stalks on the oval spores hold them out of contact with the spherical spores. This could also account for the absence of spore chains since spores which are not initially in contact are unlikely to adhere together in a special sequence on liberation.

On the basis of light microscopy, the capitellum of Mycotypha africana has been described as 'minutely punctate' and 'covered with very small spirally arranged

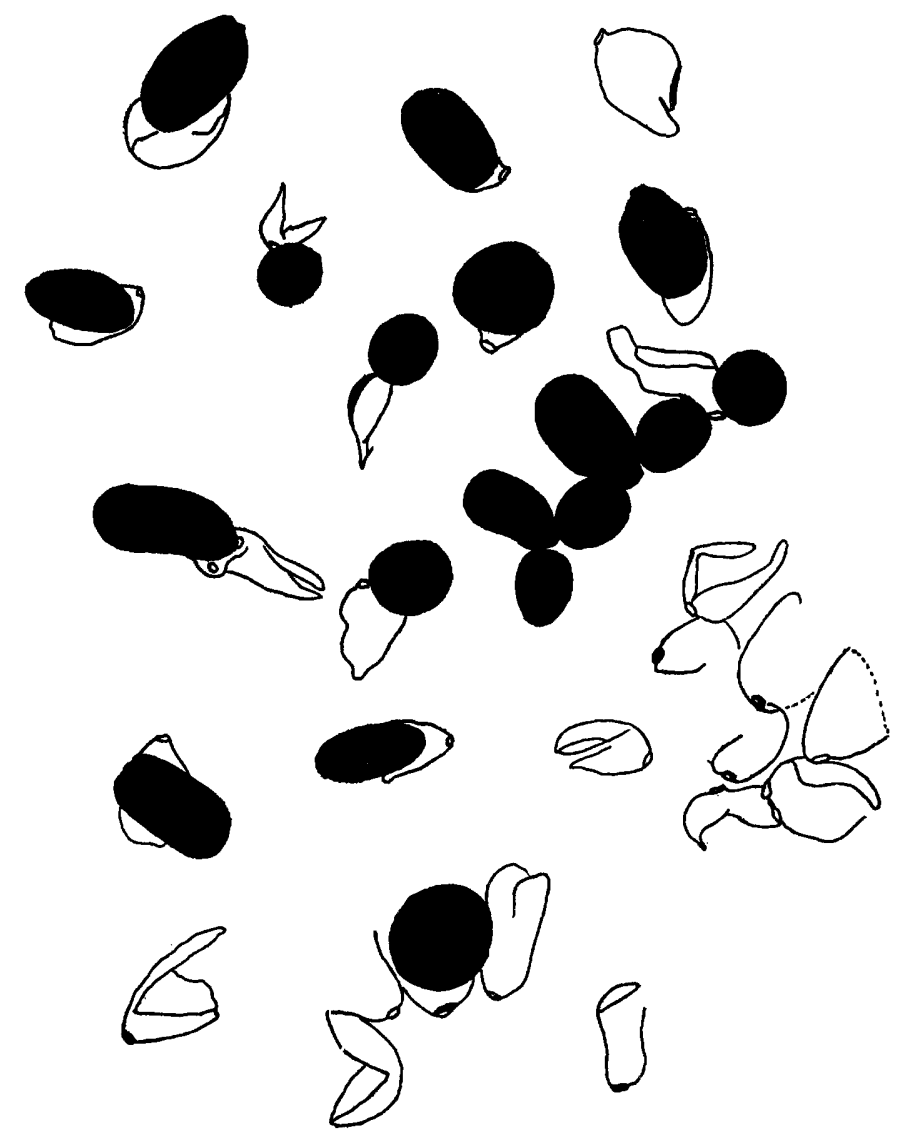

Fig. 2. Mycotypha africana: all $\times 1300$. A semi-diagrammatic representation of spores associated with separable outer membranes, traced from phase-contrast photographs. The relatively large size of some of the spores probably indicates that they were at a later stage in germination than the smaller ones.

protuberances which bear the spores' (Novak \& Backus, 1963). At high magnification under the light microscope it is possible to see the alternation of 'large' and 'small' holes at the apices of 'long' and 'short' sterigmata respectively (Pl. 3, fig. 2r). Either a shallow transverse helix or steep diagonal helices can be traced. Along one side of the capitellum illustrated, both 'long' and 'short' sterigmata can be seen. Replicas of the surface of the capitellum reveal a regular pattern of 'long' and 'short' sterigmata similar to that of $M$. microspora (P1. I, fig. I). Sterigmata are therefore dimorphic in M. africana. 
Oval spores are probably borne on the 'long' sterigmata and spherical spores on the 'short' ones. The evidence for this view is largely indirect because spores are closely packed on the capitellum (Pl. 3, fig. 20) and it is usually only possible to view sterigmata after spore liberation. However, in part of the capitellum illustrated (Pl. 3, fig. 22) two diagonal rows of spores remain attached. Alongside each row is a row of three or four 'large' holes, assumed to be apertures of 'long' sterigmata. At the edge of this capitellum (labelled A), the peripheral spore from each diagonal row looks spherical and clearly alternates with oval spores. Thus, by inference, spherical spores are attached to 'short' sterigmata. At points indicated by arrows (P1. 3, fig. 22), oval spores viewed obliquely appear to be attached to 'long' sterigmata. This view is further supported by evidence from electron micrographs of attached spores in profile (Pl. I, fig. 3, 5), replica (Pl. I, fig. 2) and from preliminary observations of ultrathin sections of the capitellum.

Spores of Mycotypha africana germinate readily on malt or potato-dextrose agar at $37^{\circ}$. Within Io to $12 \mathrm{hr}$ spores swell considerably. Both forms of spore have a relatively loose outer membrane which is shed as the germinating spore expands. Empty membranes are readily demonstrated by means of phase-contrast microscopy and appear to be common in preparations of spores allowed to germinate for approximately Io to I $2 \mathrm{hr}$. They have also been observed in preparations of ungerminated spores and in ultrathin sections. The base of a ruptured membrane remains intact and appears as a refractive circle. The circle is the region of attachment to the sterigma. The apex of the membrane is often ruptured and, in oval spores where this may be clearly observed, a longitudinal fissure runs towards the base. Ruptured membranes are illustrated (Pl. 3, fig. 23 to 32 ; Fig. 2).

\section{DISCUSSION}

Sterigmata are dimorphic in the two described Mycotypha species. Basidial dimorphism is a well-known phenomenon in Coprinus species (Buller, 1924), where long basidia constitute the first generation and short basidia the second. It seems likely therefore that a parallel system has evolved in the genus Mycotypha, where 'long' sterigmata develop first, followed by a second generation of 'short' sterigmata. This view is largely theoretical. However, in the young capitella illustrated (Pl. I, fig. 4, 8, 9), 'short' sterigmata are barely developed whereas the 'long' sterigmata are relatively well developed. This evidence appears to support the idea that 'short' sterigmata are second generation.

In contrast to Coprinus, spores in Mycotypha africana (and probably $M$. microspora) are dimorphic. In the absence of experimental evidence, a discussion of the probable significance of this phenomenon can only be speculative. One possibility is that the production of spherical and oval spores, linked with 'short' and 'long' sterigmata, may result in making full use of the space available for spore production. Spores are packed closely together on the capitellum (P1. 3, fig. 20). Secondly, it is possible that each form of spore has a different function and this aspect is under investigation.

The separable outer membrane of the spore is likely to be homologous with the sporangiole wall of such species as Cokeromyces recurvatus Poitras or Choanephora trispora (Thaxt.) Sinha. Thus, the probability is that the 'conidium' of Mycotypha africana is a single-spored sporangiole. Benjamin ( 1960 , plate $2 f$ ), demonstrated 
spores of Cokeromyces poitrasii associated with broken membranes which he interpreted as sporangiole membranes. This species produces single-spored sporangioles. The apparent similarity in form of the ruptured sporangiolar membrane to that in $M$. africana is remarkable. Further, as seen in replica, spore surfaces are similar and their capitella bear persistent sterigmata when spores are detached. Zygospores are formed between opposed progametangia in both species. It is generally considered that Cunninghamellaceae are fungi in which the conidium represents the final stage in reduction of a single-spored sporangiole since spore and sporangial walls cannot be distinguished as separate structures. The essential difference between Cunninghamellaceae and Thamnidiaceae is that in the former only conidia are produced in the asexual stage, whereas in the latter, sporangia and/or sporangioles are produced. It seems reasonable to interpret the separable outer wall of the liberated spore in $M$. africana as that of a sporangiole, in which case this species should probably be removed from Cunninghamellaceae and classified in Thamnidiaceae.

The author wishes to thank Professor C. T. Ingold for his advice and encouragement in this study; Dr J. D. Dodge for kindly reading and criticizing the manuscript and $\mathrm{Mr} \mathrm{E}$. V. Morris for assistance in preparing the photographs.

\section{REFERENCES}

Benjamin, R. K. (1960). Two new members of the Mucorales. Aliso 4, 523.

Buller, A. H. R. (1924). Researches on Fungi, vol. 3, Hafner.

FENNER, E. A. (1932). Mycotypha microspora, a new genus of the Mucoraceae. Mycologia 24, 187.

Gaumann, E. A. (1928). Comparative Morphology of Fungi. Translated and revised by C. W. Dodge. London: McGraw-Hill.

Hesseltine, C. W. (1955). Genera of Mucorales with notes on their synonymy. Mycologia 47, 344.

Novak, R. O. \& Backus, M. P. (1963). A new species of Mycotypha. with a zygosporic stage. Mycologia 55, 790.

YounG, T. W. K. (1968). Electron microscopic study of the asexual structures in Mucorales. Proc Linn. Soc. Lond. 179, 1.

\section{EXPLANATION OF PLATES}

All magnifications are approximate.

Plate I

Fig. I. Mycotypha africana. Replica of part of a capitellum showing dimorphic sterigmata. $\times 4500$.

Fig. 2. $M$. africana. Replica of two oval spores attached to 'long' sterigmata. $\times 7000$.

Fig. 3. $M$. africana. Silhouette of an oval spore attached to a 'long' sterigma. $\times 8750$.

Fig. 4. M. microspora. Replica of part of a young capitellum. 'Short' sterigmata are not well developed. $\times 7000$.

Fig. 5. M. africana. Silhouette of a spherical spore apparently attached to a 'short' sterigma. $\times 8750$.

Fig. 6. M. africana. Replica of a chain of spores. $\times 4500$.

Fig. 7. M. africana. Phase-contrast photograph of a chain of spores. $\times 1000$.

Fig. 8. $M$. microspora. Replica of the young capitellum illustrated in fig. 4. Helical rows of 'long' sterigmata are evident. 'Short' sterigmata are barely perceptible at this magnification. $\times 2200$.

Fig. 9. M. africana. Replica of a young capitellum. An apparently oval spore is attached to a 'long' sterigma. $\times 3000$.

Fig. I0. M. microspora. Some variety of spore form as seen in silhouette. $\times 2200$. 

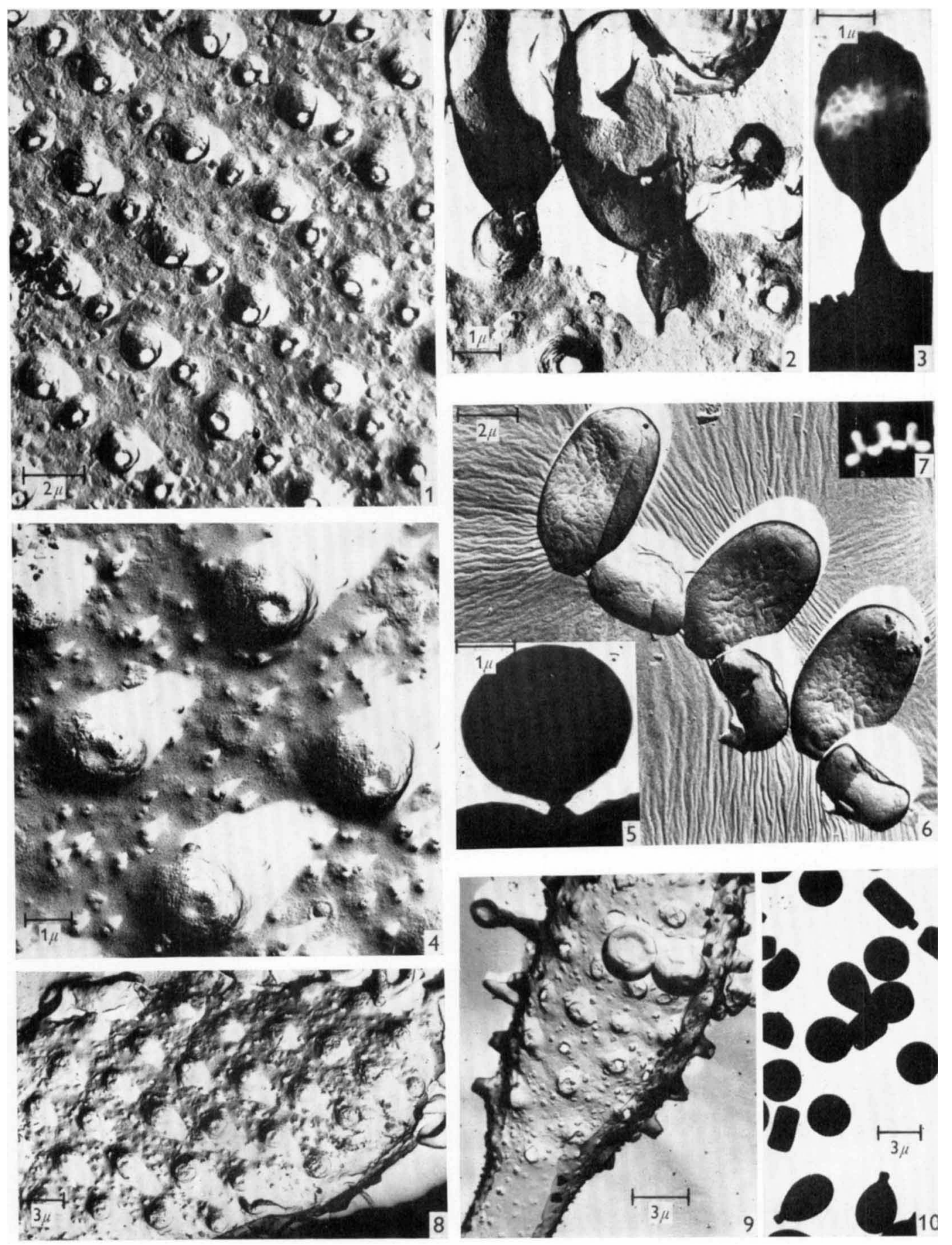
Journal of General Microbiology, Vol. 55, No. 2

Plate 2
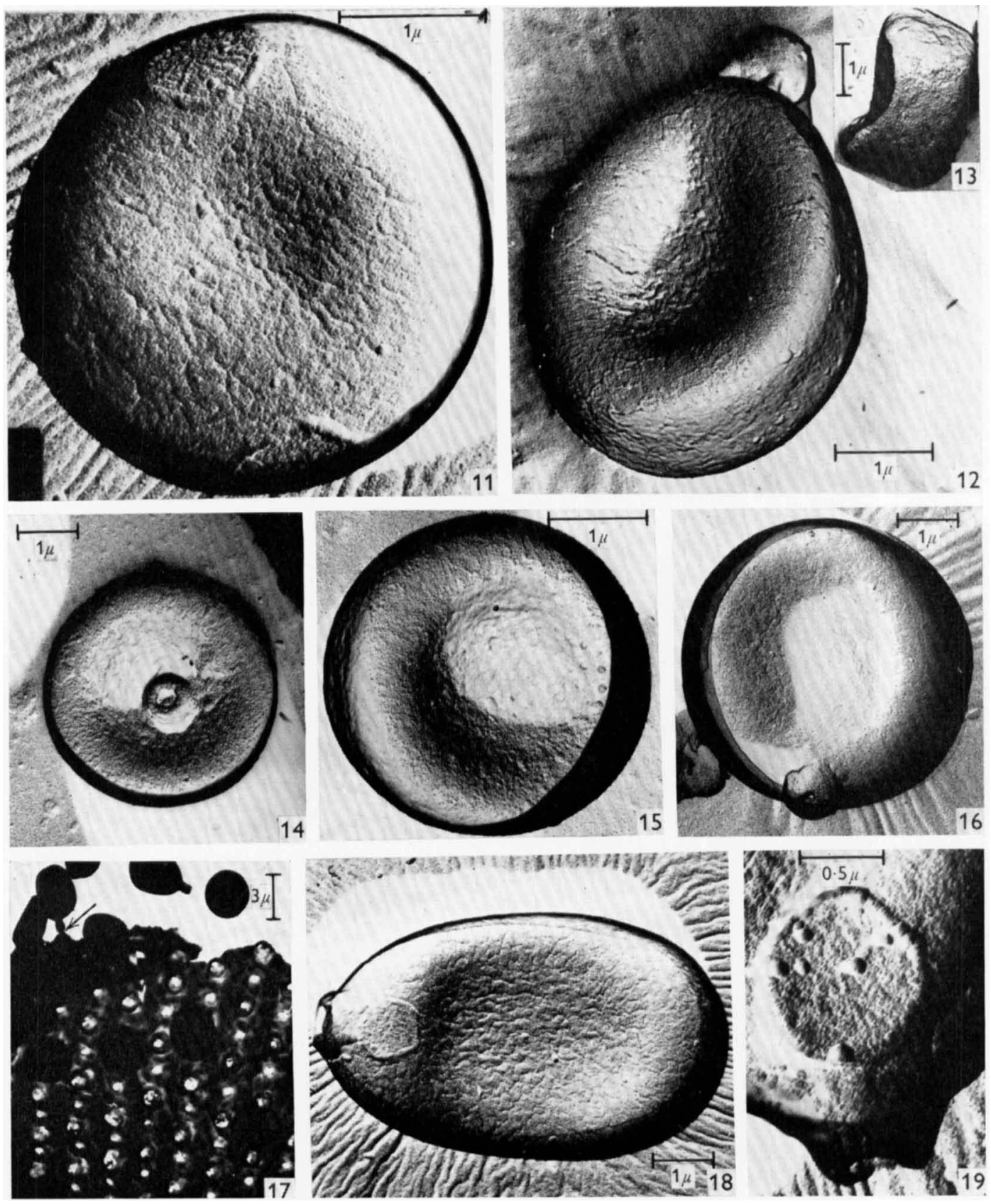

T. W. K. YOUNG 

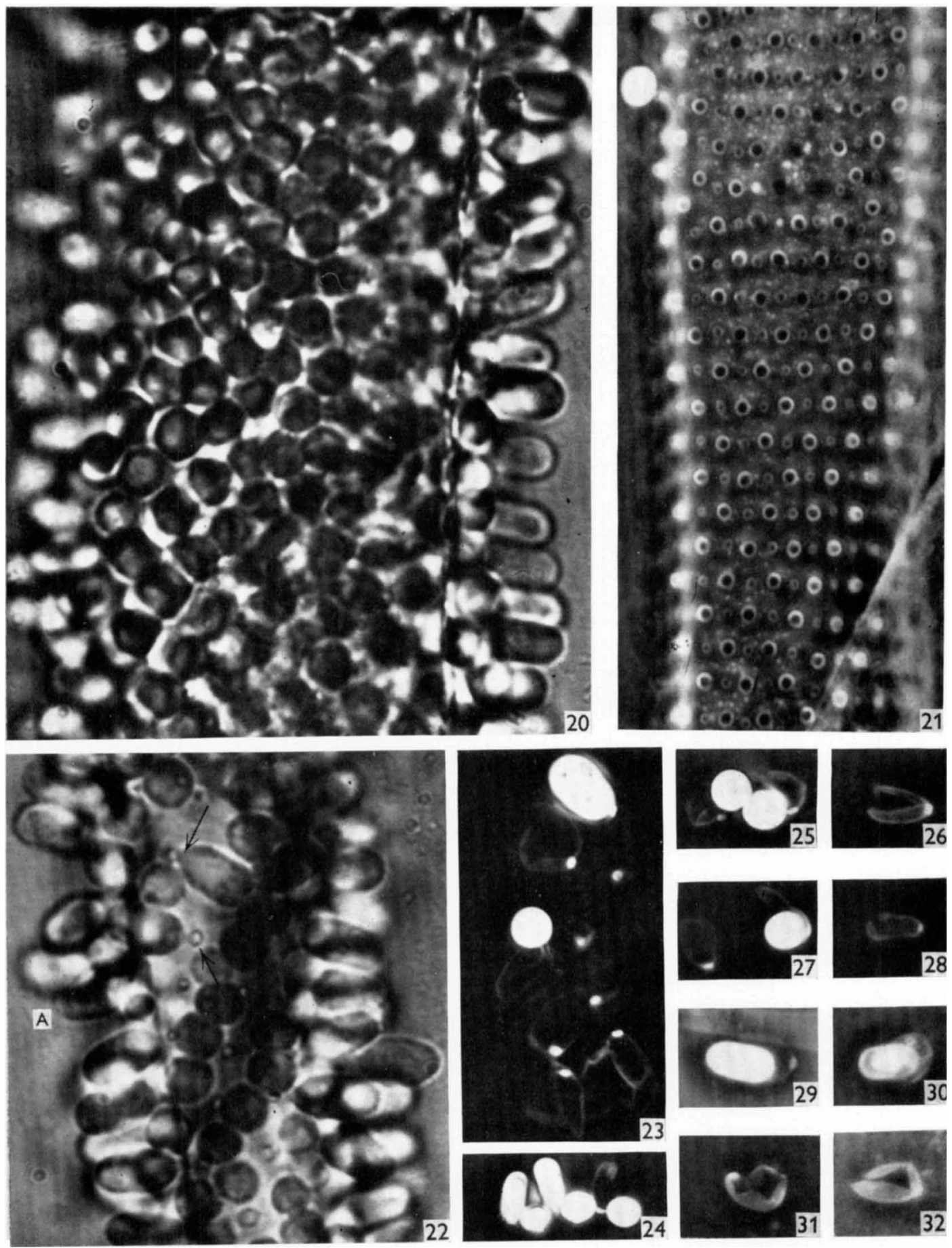

\section{(1)}
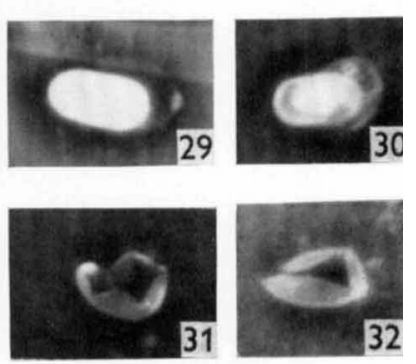

T. W. K. YOUNG 



\section{Plate 2}

Fig. I1. M. africana. Replica of a spherical spore bearing two raised areas. $\times 21,000$.

Fig. 12. M. microspora. Replica of an oval spore. $\times 14,250$.

Fig. I3. $M$. microspora. Replica of a spore. This corresponds to approximately rectangular spores seen in profile and is probably a collapsed spherical spore. $\times 7500$.

Fig. 14. M. microspora. Replica of a spherical spore with the stalk uppermost. $\times 8750$.

Fig. 15. M. microspora. Replica of a spherical spore with the stalk downwards. $\times 14,250$.

Fig. I6. M. microspora. Replica of a spherical spore with the stalk almost horizontal. $\times 8750$.

Fig. 17. M. microspora. The internal surface of part of a capitellum as seen in silhouette. The arrow indicates the annular constriction at the junction of a spore stalk and a sterigma which is still attached to the outer surface of the capitellum. $\times 2200$.

Fig. 18. M. africana. Replica of an oval spore. A raised area is visible towards the base of the spore. $\times 8750$.

Fig. 19. M. africana. Replica of a raised area towards the base of an oval spore. Note that the spore stalk is relatively short in comparison with that on the oval spore of $M$. microspora in fig. 12 . $\times 24,500$.

\section{Plate 3}

Fig. 20 and 22 were taken with ordinary light, the remainder by means of anoptral phase contrast. All of $M$. africana and $\times 2000$.

Fig. 20. Spores closely packed on the capitellum. Oval spores project well beyond the spherical ones.

Fig. 21. A capitellum from which spores have become detached. Between the apertures of dimorphic sterigmata are minute spines.

Fig. 22. Two rows of spores attached to the capitellum alternate with two rows of 'large' holes. These spores appear to be spherical. Arrows indicate two oval spores associated with 'large' holes.

Fig. 23 to 32. Empty membranes, in some cases associated with spores. Fig. 23 to 27 and 31 were observed in cultures of spores allowed to germinate for approximately il hr, the remainder in cultures 2 days or more in age. 